\title{
Internet Gaming Disorder Co-morbidity Linked to Depression and Other Affective Problems
}

\section{Trevor Archer*}

Department of Psychology, University of Gothenburg, Gothenburg, Sweden

*Corresponding author: Archer T, Department of Psychology, University of Gothenburg, Box 500, SE-430 50 Gothenburg, Sweden, Tel: +46 317864694 ; E-mail: trevor.archer@psy.gu.se

Received date: April 25, 2018; Accepted date: April 28, 2018; Published date: May 04, 2018

Copyright: @ 2018 Archer T. This is an open-access article distributed under the terms of the Creative Commons Attribution License; which permits unrestricted use; distribution; and reproduction in any medium; provided the original author and source are credited.

\section{Editorial}

The links between excessive internet game play and depressive symptoms are well-established [1,2]. Many of the problematic and dysfunctional tendencies often defined by loss of impulse control that are linked with internet/video gaming and the diagnosis of internet gaming disorder (IGD) have been shown to have connections also with other conditions, including emotional, cognitive, motivational and somatic disruptions expressed in anhedonia, anxiety, sleep disturbance and depressive states and various types of social and generalized anxiety, psychological distress, lower levels of psychological resilience and symptoms of attention-deficit hyperactivity disorder [3-7]. IGD exerts a negative impact upon physical, psychological, social, and occupational functioning within several domains of the affected individual, which often leading to severe consequences [8]. Users combining computer-games and smart phone-games were observed to exhibit a higher prevalence of IGD, depression and anxiety disorders, and substance use disorders [9]. Unsurprisingly, 89 percent of IGDdiagnosed and 92 percent of IGD-diagnosed, respectively, presented symptoms of depression and anxiety [10]. Patients presenting IGD were shown to be of younger age, with a greater likelihood of being single and unemployed, as well as presenting also a lower age-level for disorder onset [11]. Additionally, these patients expressed a lower level of somatization and depressiveness scores together with lower prevalence of tobacco use but higher food addiction scores accompanied by a higher mean body mass index. Furthermore, they evidenced lower novelty-seeking and persistence traits. Thus, the consensus of several clinical and non-clinical studies establishes that in IGD depressiveness and other affective problems, including gender, problematic alcohol use, anxiety, and a past history of psychiatric counselling or treatment due to internet gaming use, are common comorbidities [12]. These issues serve to underline the problematic nature of IGD $[13,14]$.

Suffice it to say, the gaming disorder constitutes a particular form of behavioral addiction that has been described as the individual's loss of control with persistent and recurrent use of internet gaming culminating in the marked impairment of adequate psychosocial functioning [15]. The poor quality of interpersonal relationships, as shown by impaired social support and social network, social isolation and loneliness, all serve to express the emotional underpinings of disorder [16-18]. Relationships between major depressive disorder or dysthymic disorder among college students have been reported, although there are negative findings and inconsistency [19-22]. Individuals who used internet gaming more persistently showed greater signs of depressiveness than those individuals who did not, observed from a two-year longitudinal study [23]. It has been found that the symptoms of IGD were associated positively with depression and impulsiveness and concurrently associated negatively with the quality of the afflicted individual's interpersonal relationships, the latter emphasising strong mediational influences; notably, hyperimpulsiveness was linked to greater deterioration of interpersonal relationships [24]. Among patients presenting IGD diagnosis, Bupropion, a noradrenergic-dopamine reuptake inhibitor and nicotine-receptor antagonist, induced greater therapeutic effects than the serotonin reuptake inhibitor, citalopram, upon the reduction of impulsiveness and attentional problems whereas the decreased brain connectivity between the salience network and the default mode network seems to be associated with an improvement in excessive IGD symptoms and impulse-control problems [25]. In this context, it has been observed that the co-morbidity relationship between IGD combined with alcohol use disorder presented higher levels of severe psychopathological impairments, and in these cases the respective afflicted individuals spent more money on gaming than either the IGD diagnosed or alcohol use disordered individuals by themselves [26]. IGD individuals and alcohol abusing individuals differ also with regard to attributes associated with temperament and character whereby the severity of the former correlated positively with novelty seeking score, impulsiveness and attention and the latter expressed harm avoidance score and depressed mood [27].

Alterations of regional brain integrity has been found to be compromised also: it has been observed that there was a lesser extent of gray matter density in the left dorsolateral prefrontal cortex among the IGD patient group than among the Internet gaming control group and the non-gaming control group with the gray matter density being associated with a lifetime usage of Internet gaming, depressed mood, craving, and impulsivity among the gaming users; a striatal volumetric analysis found a marked reduction in the right nucleus accumbens region in the IGD patient group and its relationship with lifetime usage of gaming and depressive symptoms; these findings imply that changes within the brain structures involved in the reward system, or pertaining to a reward deficiency syndrome, subtend a relationship with IGD-related behavioral characteristics and/or attributes [28]. In patients presenting co-morbid IGD and major depressive disorder, there was an association between reduced inter-hemispheric connectivity in the frontal region and vulnerability to attention problems implying that intra-hemisphere connectivity in the frontotemporo-parieto-occipital areas may be the consequence of excessive online gaming behaviour [29]. Procrastination, associated with the clinical severity of IGD, positively associated with depression, hostility, and impulsivity, but after controlling for depression, hostility, and impulsivity, is still linked with IGD among young adults presenting the disorder [30]. Several other affective conditions are associated with excessive gaming or computer over-usage: thus Thomee et al. [31], observed both cross-sectional and prospective relationships between computer gaming and overweight/obesity among 20 to 24 year-old Swedish young women $(n=4073)$, following adjustment for age, 
occupation, physical activity, sleep, social support, and total computer use. In a follow-up study Thomee et al. [32], it was found that sleep disturbances among male participants ( $\mathrm{n}=1458,20$ to 24 years-of-age) were associated with high-to-medium computer users as opposed to low computer users; medium computer-gaming among women users $(n=2705)$ was linked to symptoms of depression. Finally, juveniles presenting IGD displayed markedly blunted neural responses within various different subcortical and cortical brain regions such as the striatum, insula, lateral prefrontal cortex and anterior cingulate while responding to negative affective cues, as well as during emotion regulation., as confirmed by Independent component analysis identified additionally between-group differences in the engagement of a fronto-cingulo-parietal network, involving decreased engagement among the IGD juveniles in comparison with the control group [33]. The demand for treatment interventions pertaining to problems related to the use of video games has increased enormously among adolescents whom in most cases present co-morbid affective disorder pathologies [34,35].

In conclusion, the present treatise describes several affective, comorbid component disorders, most seriously depression and anxiety, that may or may not accompany the IGD disorder whether diagnosed or not. Thus, those individuals defined by chronic, compulsive video game-playing persistence and addiction display an increased incidence of major depressive disorders through which the excessive or problematic game-playing may interact with depression, anxiety and/or several affective conditions, clinically, and may magnify the impulsive behaviors associated with video gaming disorders.

\section{References}

1. Han DH, Renshaw PF (2012) Bupropion in the treatment of problematic online game play in patients with major depressive disorder. Psychopharmacol 26: 689-696.

2. Park S, Jeon HJ, Son JW, Kim H, Hong JP (2017) Correlates, comorbidities, and suicidal tendencies of problematic game use in a national wide sample of Korean adults. Int J Ment Health Syst 11: 35.

3. Griffiths MD, Nuyens F (2017) An overview of structural characteristics in problematic video game playing. Curr Addict Rep 4: 272-283.

4. Guillot CR, Bello MS, Tsai JY, Huh J, Leventhal AM, et al. (2016) Longitudinal associations between anhedonia and internet-related addictive behaviors in emerging adults. Comput Human Behav 62: 475-479.

5. Ioannidis K, Treder MS, Chamberlain SR, Kiraly F, Redden SA, et al. (2018) Problematic internet use as an age-related multifaceted problem: Evidence from a two-site survey. Addict Behav 81: 157-166.

6. Park JH, Han DH, Kim BN, Cheong JH, Lee YS (2016) Correlations among social anxiety, self-esteem, impulsivity, and game genre in patients with problematic online game playing. Psychiatry Investig 13: 297-304.

7. Wu AMS, Chen JH, Tong KK, Yu S, Lau JTF (2018) Prevalence and associated factors of Internet gaming disorder among community dwelling adults in Macao, China. J Behav Addict 7: 62-69.

8. Naskar S, Victor R, Nath K, Sengupta C (2016) "One level more:" A narrative review on internet gaming disorder. Ind Psychiatry J 25: 145-154.

9. Paik SH, Cho H, Chun JW, Jeong JE, Kim DJ (2017) Gaming device usage patterns predict internet gaming disorder: Comparison across different gaming device usage patterns. Int J Environ Res Public Health 14: E1512.

10. González-Bueso V, Santamaría JJ, Fernández D, Merino L, Montero E, et al. (2018) Association between internet gaming disorder or pathological video-game use and comorbid psychopathology: A comprehensive review. Int J Environ Res Public Health 15: E668.

11. Mallorquí-Bagué N, Fernández-Aranda F, Lozano-Madrid M, Granero R, Mestre-Bach G, et al. (2018) Internet gaming disorder and online gambling disorder: Clinical and personality correlates. J Behav Addict 6: 669-677.

12. Wang HR, Cho H, Kim DJ (2018) Prevalence and correlates of comorbid depression in a nonclinical online sample with DSM-5 internet gaming disorder. J Affect Disord 226: 1-5.

13. Archer T, Wentz K (2017) Internet-video gaming: symptoms, epidemiology, neurophysiology and interventional aspects. J Child Adolesc Behav 5: 345.

14. Archer T, Garcia D, Moradi S (2016) The enigmatic influence of videointernet gaming: liabilities and assets over the lifespan. Sports Med Rehabil J 1: 1008-1017.

15. American Psychiatric Association (2013) Diagnostic and Statistical Manual of Mental Disorders (DSM- $5^{\circ}$ ) (5th edn). American Psychiatric Association, Washington, DC, USA.

16. Chen KH, Oliffe JL, Kelly MT (2018) Internet gaming disorder: An emergent health issue for men. Am J Mens Health.

17. Kim J, LaRose R, Peng W (2009) Loneliness as the cause and the effect of problematic internet use: The relationship between internet use and psychological well-being. Cyberpsychol Behav12: 451-455.

18. Yao MZ, Zhong ZJ (2014) Loneliness, social contacts and internet addiction: A cross-lagged panel study. Comput Hum Behav 30: 164-170.

19. Hagerty BM, Williams RA (1999) The effects of sense of belonging, social support, conflict, and loneliness on depression. Nurs Res 48: 215-219.

20. Ko CH, Yen JY, Chen CS, Chen CC, Yen CF (2008) Psychiatric comorbidity of internet addiction in college students: An interview study. CNS Spectr 13: 147-153.

21. Bahrainian SA, Alizadeh KH, Raeisoon MR, Gorji OH, Khazaee A (2014) Relationship of internet addiction with self-esteem and depression in university students. J Prev Med Hyg 55: 86-89.

22. Dieris-Hirche J, Bottel L, Bielefeld M, Steinbüchel T, Kehyayan A, et al. (2017) Media use and internet addiction in adult depression: A casecontrol study. Comput Hum Behav 68: 96-103.

23. Ko CH, Yen JY, Chen CS, Yeh YC, Yen CF (2009) Predictive values of psychiatric symptoms for internet addiction in adolescents: A 2-year prospective study. Arch Pediatr Adolesc Med 163: 937-943.

24. Ryu H, Lee JY, Choi A, Park S, Kim DJ, et al. (2018) The relationship between impulsivity and internet gaming disorder in young adults: Mediating effects of interpersonal relationships and depression. Int J Environ Res Public Health 15: E458.

25. Nam B, Bae S, Kim SM, Hong JS, Han DH (2017) Comparing the effects of bupropion and escitalopram on excessive internet game play in patients with major depressive disorder. Clin Psychopharmacol Neurosci 15: 361-368.

26. Na E, Lee H, Choi I, Kim DJ (2017) Comorbidity of internet gaming disorder and alcohol use disorder: A focus on clinical characteristics and gaming patterns. Am J Addict 26: 326-334.

27. Lee YS, Son JH, Park JH, Kim SM, Kee BS, et al. (2017) The comparison of temperament and character between patients with internet gaming disorder and those with alcohol dependence. J Ment Health 26: 242-247.

28. Choi J, Cho H, Kim JY, Jung DJ, Ahn KJ, et al. (2017) Structural alterations in the prefrontal cortex mediate the relationship between Internet gaming disorder and depressed mood. Sci Rep 7: 1245.

29. Youh J, Hong JS, Han DH, Chung US, Min KJ, et al. (2017) Comparison of Electroencephalography (EEG) coherence between Major Depressive Disorder (MDD) without comorbidity and MDD comorbid with internet gaming disorder. J Korean Med Sci 32: 1160-1165.

30. Yeh YC, Wang PW, Huang MF, Lin PC, Chen CS, et al. (2017) The procrastination of Internet gaming disorder in young adults: The clinical severity. Psychiatry Res 254: 258-262.

31. Thomée S, Härenstam A, Hagberg M (2012) Computer use and stress, sleep disturbances, and symptoms of depression among young adults-a prospective cohort study. BMC Psychiatry 12: 176.

32. Thomée S, Lissner L, Hagberg M, Grimby-Ekman A (2015) Leisure time computer use and overweight development in young adults-a prospective study. BMC Public Health 15: 839. 
Citation: Trevor Archer (2018) Internet Gaming Disorder Co-morbidity Linked to Depression and Other Affective Problems. Clin Depress 4: e107. doi:10.4172/2572-0791.1000e107

Page 3 of 3

33. Yip SW, Gross JJ, Chawla M, Ma SS, Shi XH, et al. (2018) Is neural processing of negative stimuli altered in addiction independent of drug effects? Findings from drug-naïve youth with internet gaming disorder. Neuropsychopharmacology 43: 1364-1372.

34. Martín-Fernández M, Matalí JL, García-Sánchez S, Pardo M, Lleras M, et al. (2016) Adolescents with Internet Gaming Disorder (IGD): Profiles and treatment response. Adicciones 29: 125-133.
35. Han DH, Kim SM, Bae S, Renshaw PF, Anderson JS (2016) A failure of suppression within the default mode network in depressed adolescents with compulsive internet game play. J Affect Disord 194: 57-64. 